Article

\title{
Structurally Complex Frank-Kasper Phases and Quasicrystal Approximants: Electronic Origin of Stability
}

\author{
Valentina F. Degtyareva * and Natalia S. Afonikova \\ Institute of Solid State Physics, Russian Academy of Sciences, Chernogolovka 142432, Russia; natasha@issp.ac.ru \\ * Correspondence: degtyar@issp.ac.ru
}

Academic Editor: Dmitry A. Shulyatev

Received: 11 November 2017; Accepted: 1 December 2017; Published: 4 December 2017

\begin{abstract}
Metal crystals with tetrahedral packing are known as Frank-Kasper phases, with large unit cells with the number of atoms numbering from hundreds to thousands. The main factors of the formation and stability of these phases are the atomic size ratio and the number of valence electrons per atom. The significance of the electronic energy contribution is analyzed within the Fermi sphere-Brillouin zone interaction model for several typical examples: $\mathrm{Cu}_{4} \mathrm{Cd}_{3}, \mathrm{Mg}_{2} \mathrm{Al}_{3}$ with over a thousand atoms per cell, and for icosahedral quasicrystal approximants with 146-168 atoms per cell. Our analysis shows that to minimize the crystal energy, it is important that the Fermi sphere (FS) is in contact with the Brillouin zones that are related to the strong diffraction peaks: the zones either inscribe the FS or are circumscribed by the FS creating contact at edges or vertices.
\end{abstract}

Keywords: Frank-Kasper phases; icosahedral quasicrystal approximants; Hume-Rothery electron concentration rule

\section{Introduction}

Common metallic structures are based on high-symmetry atomic cells, such as face-centered cubic $(f c c)$, close-packed hexagonal $(h c p)$, and body-centered cubic $(b c c)$, that have a coordination number of either 12 or $8+6$. These structures are found in elements in their metallic phases; they are also formed in binary alloys and compounds if constituent elements have small differences in atomic size and electronegativity. A special family of intermetallic alloys is based on tetrahedrally-packed phases, called Frank-Kasper phases, where tetrahedra form polyhedra with coordination numbers of 12 (icosahedron), 14, 15, and 16 [1]. Frank-Kasper polyhedra are basic structural units for many types of metallic alloys, including very complex structures with the number of atoms in the unit cell up to hundreds or thousands, as well as quasicrystals. Structures and properties of complex metallic alloys are considered in several books and review papers [2-7].

Crystal structures of very complex intermetallics are built with successive shells of atoms, including polyhedra, like icosahedrons, dodecahedrons, rhombic triacontahedrons, and truncated icosahedrons (soccer ball or "fullercage" [8]). These atomic arrangements are called "Russian doll" ("matryoshka") clusters [9]. Similar atomic packings exist in icosahedral quasicrystals, known as Mackay-type, Bergman-type, and Tsai-type. These clusters build the structures of quasicrystal approximants with the periodic arrangements in the $b c c$ cell $(1 / 1$ type) or more complex periodic structures.

Quasicrystal and approximant phases exist at certain alloy compositions usually defined by valence electron concentrations and represent a kind of Hume-Rothery phases [2-5]. The crystal energy is lowered by contact of the Fermi level to the Brillouin zone planes formed by strong diffraction peaks. Historically, Hume-Rothery phases were considered first in the $\mathrm{Cu}-\mathrm{Zn}$ alloys and related binary systems of $\mathrm{Cu}$-group elements with the neighboring elements of higher valences [10-12]. 
Classical Hume-Rothery phases with $f c c \rightarrow b c c \rightarrow$ complex $\gamma$-brass $\rightarrow h c p$ are defined by the number of valence electrons per atom, such as $1.35 \rightarrow 1.5 \rightarrow 1.62 \rightarrow 1.75$ [10]. In the case of quasicrystal approximants, these values are usually higher. Diffraction patterns of these compounds consist of several strong diffraction peaks that should be taken into account when considering the stability of their crystal structures.

In the present paper the model of the Fermi-sphere-Brillouin zone (FS-BZ) interaction is applied to complex structures, considering the FS inscribed into the BZ, as well as the FS enveloped inner zones contacting the edges or vertices. Both cases of FS-BZ configuration should affect the band-structure energy and decrease the crystal energy.

\section{Theoretical Background and Method of Analysis}

Formation of binary compounds at a certain alloy composition is defined by some important factors characterizing the alloy constituents, such as the difference in atomic sizes, electronegativity etc. Beyond these factors, formation of metallic structures is defined by effects of the Fermi sphere-Brillouin zone (FS-BZ) interaction. The Hume-Rothery mechanism has been identified to play a role in the stability of structurally-complex alloy phases, quasicrystals, and their approximants [2,13-16]. Formation of the complex structures of elemental metals under pressure can also be related to the Hume-Rothery mechanism [17-19]. Phase diagrams of binary alloys, such as Au-Cd represent several phases with complex structures that follow the Hume-Rothery rule of stability at certain electron concentrations [20].

The band structure contribution to the crystal structure energy can be estimated by analyzing configurations of Brillouin-Jones zone planes within the nearly free-electron model. A special program, BRIZ, has been developed [21] to construct FS-BZ configurations and to estimate some parameters, such as the Fermi sphere radius $\left(\mathrm{k}_{\mathrm{F}}\right)$, values of reciprocal wave vectors of $B Z$ planes $\left(\mathrm{q}_{\mathrm{hkl}}\right)$, and volumes of $\mathrm{BZ}$ and FS. The BZ planes are selected to match the condition $\mathrm{q}_{\mathrm{hkl}} \approx 2 \mathrm{k}_{\mathrm{F}}$, which has a significant structure factor. In this case an energy gap is opened on the BZ plane leading to the lowering of the electron band energy. The ratio of $\frac{1}{2} q_{h k l}$ to $k_{F}$ is usually less than 1 , and equal to $\sim 0.95$; it is called a "truncation" factor. In the FS-BZ presentations by the BRIZ program the BZ planes cross the FS whereas, in the real system, the Fermi sphere is deformed and accommodated inside the BZ due to an increase of the electron density and to a decrease in the electron energy near the BZ plane.

The crystal structure of a phase chosen for the analysis by the BRIZ program is characterized by the lattice parameters and the number of atoms in the unit cell, which define the average atomic volume $\left(\mathrm{V}_{\mathrm{at}}\right)$. The valence electron concentration $(\mathrm{z})$ is the average number of valence electrons per atom that gives the value of the Fermi sphere radius $\mathrm{k}_{\mathrm{F}}=\left(3 \pi^{2} \mathrm{z} / \mathrm{V}_{\mathrm{at}}\right)^{1 / 3}$. Further structure characterization parameters are the number of BZ planes that are in contact with the FS, the degree of the "truncation" factor, and the value of BZ filling by electronic states, defined as a ratio of the volumes of FS and BZ. Presentations of the FS-BZ configurations are given with the orthogonal axes with the following directions in the common view: $\mathrm{a}^{*}$ is looking forward, $\mathrm{b}^{*}$ to the right and $\mathrm{c}^{*}$ upward.

The FS-BZ interaction for relatively simple structures, such as $\mathrm{Cu}-\mathrm{Zn}$ phases, is usually described as a contact of the FS to the BZ planes resulting in the decrease of the electronic energy by the formation of the energy gap. In the case of complex structures, diffraction patterns consist of several strong reflections at wave vectors well below $2 \mathrm{k}_{\mathrm{F}}$, forming BZs that are inscribed by the FS with contacts at the edges or vertices. These cases are constructed with the BRIZ program by proper choice of presentations: "sphere inside polyhedron" or "polyhedron inside sphere". Structural stabilization due to the electronic band contribution where FS is enveloping the BZ was discussed for phase stability in In and Sn alloys [22-24] and for fcc-based phases in simple metals [25].

Significant arguments for the stability of tetrahedrally-packed structures are related to electrostatic (Ewald or Madelung) contributions to the crystal energy because of the high packing density of atoms in Frank-Kasper polyhedra. These polyhedra are forming interpenetrating building blocks that are packed in the long-range structure with a high symmetry, as, for example, in $b c c$ in the case of 
$1 / 1$ approximants. The electrostatic energy of ion-ion interactions is usually defined by sums in the real and the reciprocal space providing quick convergence [26-28]. In this relation, the configuration of BZs formed by strong reflections and accommodated inside the FS is responsible for the contribution to the Ewald energy and usually prefers high-symmetry polyhedra. The $o C 16$ structure observed in some metals and binary alloys under pressure should be mentioned as an example [29].

\section{Results and Discussion}

In this work, two groups of complex intermetallic phases are selected: with very large unit cells containing above a thousand atoms, and quasicrystal 1/1 approximants of the Bergman-type and Tsai-type. For our consideration we selected compounds with $s$ and $s p$ valence electron elements (non-transition elements) to allow us to calculate definitely the valence electron concentration. Structural data for compounds are given in Table 1. X-ray diffraction patterns and constructed Brillouin-Jones zones for these structures are presented in Figures 1-3. Crystal structure descriptions are given following the Pearson notation [30].

Table 1. Structure parameters of several metallic compounds with giant unit cells and quasicrystal approximants. Fermi sphere radius $\mathrm{k}_{\mathrm{F}}$, ratios of $\mathrm{k}_{\mathrm{F}}$ to the distances of the Brillouin zone planes $\frac{1}{2} \mathrm{q}_{\mathrm{hkl}}$, and the filling degree of the Brillouin zones by electron states $V_{F S} / V_{B Z}$ are calculated by the program BRIZ [21].

\begin{tabular}{|c|c|c|c|c|c|c|}
\hline Phase & $\mathrm{Cu}_{4} \mathrm{Cd}_{3}$ & $\mathrm{Mg}_{28} \mathrm{Al}_{45}$ & $\mathrm{CaCd}_{6}$ & $\mathrm{Al}_{30} \mathrm{Mg}_{40} \mathrm{Zn}_{30}$ & $\mathrm{Al}_{5} \mathrm{CuLi}_{3}$ & $\mathrm{Au}_{15} \mathrm{Cd}_{23} \mathrm{Zn}_{11}$ \\
\hline Pearson symbol & cF1124 & $c F 1168$ & cI168 & cI162 & cI160 & cI146 \\
\hline \multicolumn{7}{|c|}{ Structural data } \\
\hline Space group & $F \overline{4} 3 m$ & $F d \overline{3} m$ & $\operatorname{Im} \overline{3}$ & $\operatorname{Im} \overline{3}$ & $\operatorname{Im} \overline{3}$ & $\operatorname{Im} \overline{3}$ \\
\hline Lattice parameters $(\AA)$ & $a=25.871$ & $a=28.24$ & $a=15.680$ & $a=14.355$ & $a=13.891$ & $a=13.843$ \\
\hline $\mathrm{V}_{\text {at. }}\left(\AA^{3}\right)$ & 15.67 & 19.28 & 22.95 & 18.26 & 16.75 & 18.17 \\
\hline References & {$[31]$} & {$[32,33]$} & {$[34,35]$} & {$[36,37]$} & {$[38,39]$} & [14] \\
\hline \multicolumn{7}{|c|}{ FS-BZ data from the BRIZ program } \\
\hline $\begin{array}{l}\mathrm{z} \text { (number of valence } \\
\text { electrons per atom) }\end{array}$ & 1.43 & 2.62 & 2 & 2.3 & 2.18 & 1.69 \\
\hline $\mathrm{k}_{\mathrm{F}}\left(\AA^{-1}\right)$ & 1.401 & 1.590 & 1.372 & 1.551 & 1.568 & 1.403 \\
\hline $\begin{array}{l}\text { Total number } \\
\text { BZ planes }\end{array}$ & 84 & 96 & 96 & 84 & 96 & 42 \\
\hline hkl: $k_{F} /\left(\frac{1}{2} q_{h k l}\right)$ & $\begin{array}{l}(880): 1.020 \\
(955): 1.008 \\
(10.44): 1.004 \\
(11.33): 0.979\end{array}$ & $\begin{array}{l}(14.20): 1.011 \\
(10.86) \\
(10.10 .2): 1.001\end{array}$ & $\begin{array}{l}(631): 1.010 \\
(543): 0.968 \\
(701) \\
(550)\end{array}$ & $\begin{array}{l}(543): 1.002 \\
(701) \\
(550)\end{array}$ & $\begin{array}{l}(631): 1.022 \\
(543): 0.988 \\
(710)\end{array}$ & $\begin{array}{l}(503): 1.060 \\
(600): 1.030 \\
(532): 1.003\end{array}$ \\
\hline$V_{F S} / V_{B Z}$ & 0.955 & 0.966 & 0.950 & 0.945 & 0.936 & 1.00 \\
\hline
\end{tabular}

\subsection{Giant Unit Cell Compounds $\mathrm{Cu}_{4} \mathrm{Cd}_{3}$ and $\mathrm{Mg}_{2} \mathrm{Al}_{3}$}

Complex compounds with more than 1000 atoms in the unit cell were found in $\mathrm{Cu}_{4} \mathrm{Cd}_{3}$ and in $\mathrm{Mg}_{2} \mathrm{Al}_{3}$ by Samson [31,32]. Interestingly, the $\mathrm{Cu}_{4} \mathrm{Cd}_{3}$ compound differs considerably from isoelectronic phases in neighboring systems of $\mathrm{Cu}-\mathrm{Zn}$ and $\mathrm{Au}-\mathrm{Cd}$ that have, in this composition, either $b c c$ or ordered CsCl-type structures [20]. The decisive factor for this difference is that the atomic size ratio does not exceed $10 \%$ for $\mathrm{Cu} / \mathrm{Zn}$ or $\mathrm{Au} / \mathrm{Cd}$, but is $\sim 20 \%$ for $\mathrm{Cu} / \mathrm{Cd}$. Therefore, in $\mathrm{Cu}_{4} \mathrm{Cd}_{3}$, instead of the simple $b c c$ or $\mathrm{CsCl}$ structures, a tetrahedrally-packed complex compound is formed.

The diffraction pattern of $\mathrm{Cu}_{4} \mathrm{Cd}_{3}-c F 1124$ consists of diffraction peaks with relatively strong intensity grouped near the $2 \mathrm{k}_{\mathrm{F}}$ position (Figure 1a). Below the diffraction pattern, FS-BZ constructions are shown for separate peaks and for the final BZ, including 84 planes in close contact to the FS (lower-right). This consideration confirms the influential role of the Hume-Rothery mechanism for the stability of a complex compound along with an atomic size factor.

A similar approach that considers Brillouin zones in the discussion of tetrahedrally-packed structures is given in [14]. It is necessary to note that, for the $\mathrm{Cu}_{4} \mathrm{Cd}_{3}$ compound, the same group of diffraction peaks were selected for the BZ construction (see [14], Figure 40), however, the BZ form is slightly different from the construction made with the BRIZ program more accurate, as given in Figure 1a (lower-right). 


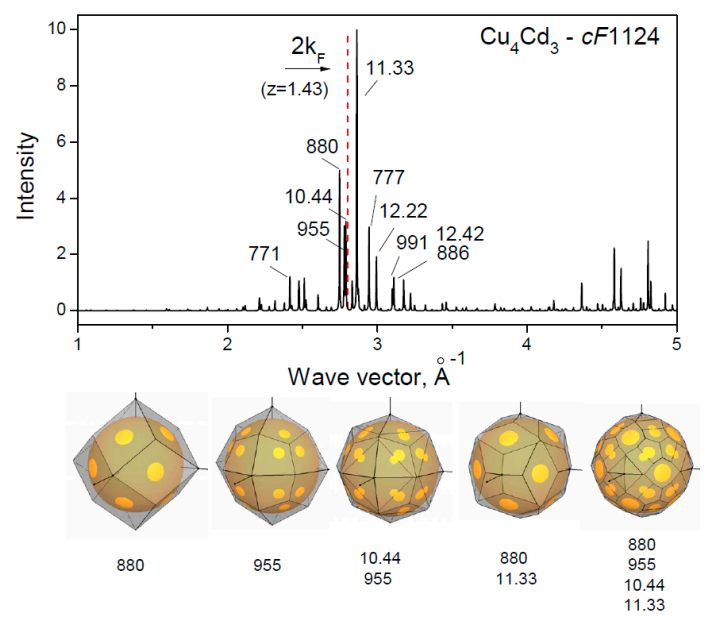

(a)

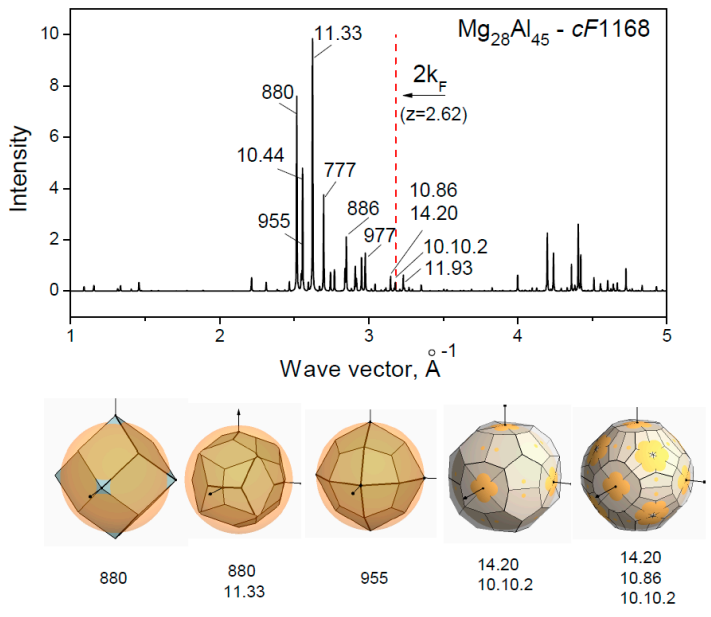

(b)

Figure 1. Calculated diffraction patterns for complex intermetallic phases (upper panel) and corresponding Brillouin-Jones zones with the Fermi spheres (lower panel). The position of $2 \mathrm{k}_{\mathrm{F}}$ for a given valence electron number per atom $\mathrm{z}$ and the $\mathrm{hkl}$ indices of the planes used for the $\mathrm{BZ}$ construction are indicated on the diffraction patterns. (a) $\mathrm{Cu}_{4} \mathrm{Cd}_{3}-c F 1124$; and (b) $\mathrm{Mg}_{28} \mathrm{Al}_{45}-c F 1168$. Structural data and FS-BZ evaluation data are given in Table 1.

The complex compound $\mathrm{Mg}_{2} \mathrm{Al}_{3}$ has a similar structure, with 1168 atoms in the unit cell, as was defined by Samson [32] and re-determined recently [33]. Structural data for this phase are given in Table 1 designated as $\mathrm{Mg}_{28} \mathrm{Al}_{45}$ following the Pauling file data [40]. The diffraction pattern for this phase (Figure $1 b$ ) is similar to that for $\mathrm{Cu}_{4} \mathrm{Cd}_{3}$ (Figure 1a) with the same group of strong reflections. However, for $\mathrm{Mg}_{28} \mathrm{Al}_{45}$ the position of $2 \mathrm{k}_{\mathrm{F}}$ for $\mathrm{z}=2.62$ falls well above the position of strong reflections. This case was discussed by Dubois with the suggestion that, for this compound, "the Fermi sphere overlaps the Jones zone" (see [7], Figure 4). Overlapping Brillouin-Jones zones by the FS for strong reflections are shown in Figure $1 \mathrm{~b}$ (lower-left, middle). Near the position of $2 \mathrm{k}_{\mathrm{F}}$ there is a group of reflections forming BZ planes in contact with the inscribed FS (lower-right).

\subsection{Quasicrystal Approximants}

Interesting groups of tetrahedrally-packed phases represent quasicrystal approximants of the 1/1-type and other types. Some of these phases have been known long before the discovery of quasicrystals: for example, $\mathrm{Mg}_{32}(\mathrm{Zn}, \mathrm{Al})_{49}$ and $\mathrm{CaCd}_{6}$ [34,36], and later they were assigned as approximants of icosahedral quasicrystals (QCs). Diffraction patterns of QCs and their approximants have strong reflections at nearly the same positions and both groups of phases exist at very close alloy 
compositions that can be estimated as valence electron concentrations (z). It is commonly assumed that QCs and their approximants are stabilized by the Hume-Rothery mechanism [2-5] and exist in regions of $\mathrm{z}$ equal to $\sim 1.8,2$, and $\sim 2.2-2.3$. For those $\mathrm{z}$ values, representative QCs-approximants are considered with the structural data listed in Table 1.

\subsubsection{Approximants $c I 168$ and $c I 162$}

Diffraction patterns for QCs-approximants $\mathrm{CaCd}_{6}-c I 168$ and $\mathrm{Al}_{30} \mathrm{Mg}_{40} \mathrm{Zn}_{30}-\mathrm{cI} 162$ and FS-BZ configurations are shown in Figure 2. Both phases have similar groups of strong reflections, whereas the $2 \mathrm{k}_{\mathrm{F}}$ positions are different and are close to the diffraction peak (631) for $\mathrm{z}=2$ and to the diffraction peaks (5 4 3), (7 10$),(550)$, for $\mathrm{z}=2.3$ (Figure 2a,b, respectively).

Strong reflections at lower wave vector values form highly-symmetrical polyhedra that are enveloped by the FS, creating a contact at the edges of the polyhedron with planes $(503)$ or vertices of the polyhedron with planes (5 3 2), (6 00 0). Due to space group symmetry $\operatorname{Im} \overline{3}$ there is a difference in the intensity for reflections within the same (hkl) set. For example, the structure $\mathrm{CaCd}_{6}-\mathrm{cI} 168$ results in an intensity ratio of $\sim 6$ for reflections (5 03 ) and (5 30 ). Assuming that only the (5 03 ) reflections contribute to the constructions of the $\mathrm{BZ}$, the resulting polyhedron is of the pentagonal dodecahedron type (Figure 2a, lower-left). The BZ polyhedron for (5 32 2) and (6 000$)$ reflections represents a rhombic triacontahedron (Figure 2a, lower-middle). Both kinds of polyhedra are inscribed into the FS and with contacts at the vertices. Thus, the polyhedra in reciprocal space are related to polyhedra in real space formed by atom clusters. It should be noted that the BZ that is in contact with the inscribed FS for $\mathrm{CaCd}_{6}$ consists mainly of (6 31 1) and (3 61 ) with nearly the same intensity (Figure 2a, lower-right).

For the approximant $\mathrm{Al}_{30} \mathrm{Mg}_{40} \mathrm{Zn}_{30}-c I 162$ the next diffraction peak with indices (5 43 ), (7 10 ), and (5 50 ) forms the BZ planes in essential contact to the FS, as shown in Figure $2 b$ (lower-right). There are 84 facets in the BZ contacting the FS at the same distance. The filling of the BZ by electron states is 0.945 (see Table 1) which satisfies the Hume-Rothery rule well. The same BZ-FS configuration for the $\mathrm{Al}_{30} \mathrm{Mg}_{40} \mathrm{Zn}_{30}$-approximant was considered in Figure 1 [37] with discussion of matching $2 \mathrm{k}_{\mathrm{F}}$ to the wave vectors of reflections (5 43$),(710)$, and (5 50 ). In addition to this, it is also necessary to consider the BZs constructed with the reflections (5 03 ) and (5 32 2)/(6 000$)$ that are enveloped by the FS, as shown in Figure $2 b$ (lower-left and middle).

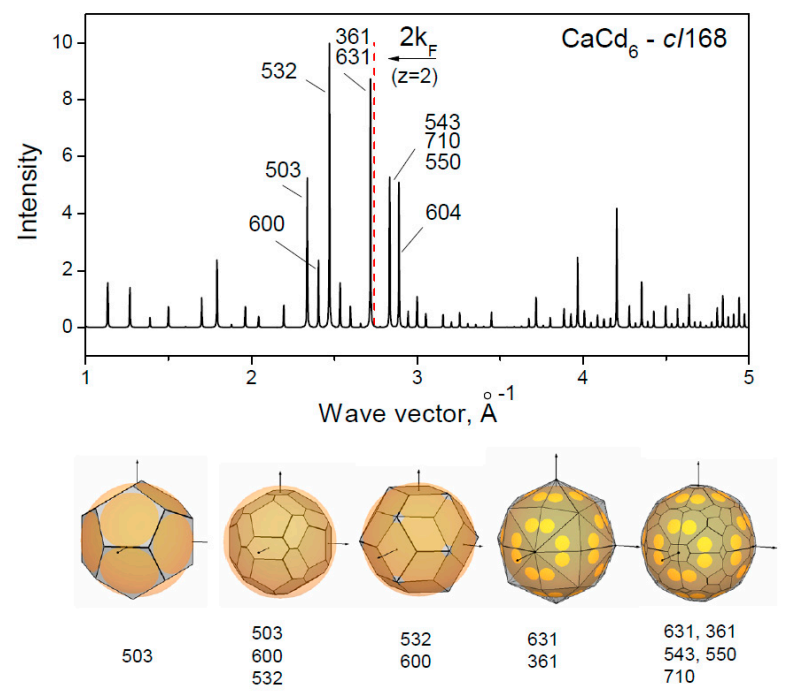

(a)

Figure 2. Cont. 


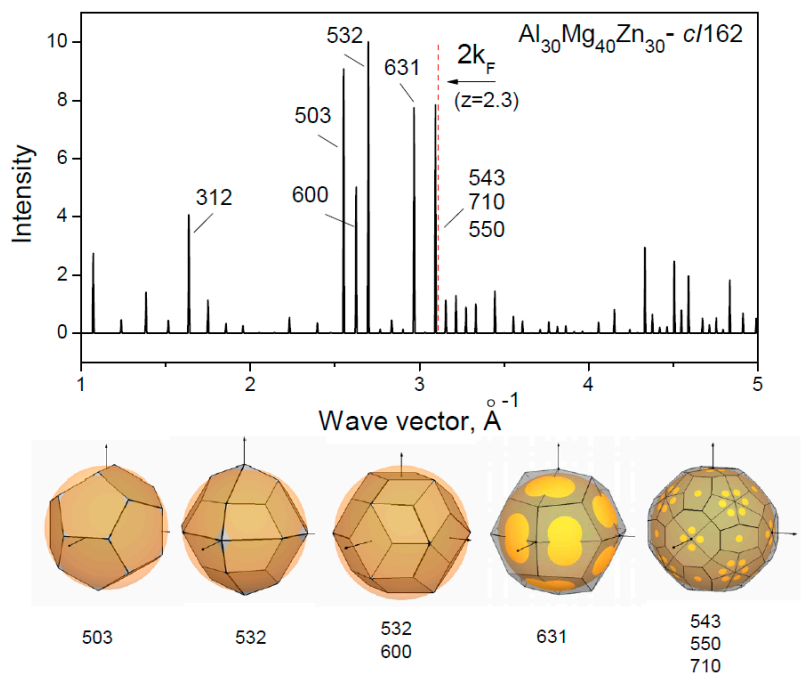

(b)

Figure 2. Calculated diffraction patterns for quasicrystal approximants (upper panel) and corresponding Brillouin-Jones zones with the Fermi spheres (lower panel). The position of $2 k_{F}$ for a given valence electron number per atom $z$ and the hkl indices of the planes used for the BZ construction are indicated on the diffraction patterns. (a) $\mathrm{CaCd}_{6}-\mathrm{cI} 168$; and (b) $\mathrm{Al}_{30} \mathrm{Mg}_{40} \mathrm{Zn}_{30}-\mathrm{cI} 162$. Structural data and FS-BZ evaluation data are given in Table 1.

\subsubsection{Approximants $c I 160$ and $c I 146$}

$\mathrm{Al}_{30} \mathrm{Mg}_{40} \mathrm{Zn}_{30}-\mathrm{cI} 162$ and $\mathrm{Al}_{5} \mathrm{CuLi}_{3}-c I 160$ have much similarity in their crystal structures, both belonging to the Bergman-type approximants. Diffraction patterns for both phases are similar, as can be seen from Figures $2 \mathrm{~b}$ and $3 \mathrm{a}$. The only difference is in the $2 \mathrm{k}_{\mathrm{F}}$ position: for $\mathrm{Al}_{5} \mathrm{CuLi}_{3}$ it is close to the reflection (6 31 ), while for $\mathrm{Al}_{30} \mathrm{Mg}_{40} \mathrm{Zn}_{30}$ it is close to reflections (5 43 ), (7 10 ), and (5 50 ), as shown in Figures $2 \mathrm{~b}$ and $3 \mathrm{a}$ (lower-right), respectively. It should be noted that, because of space group $\operatorname{Im} \overline{3}$, the intensity of the $\left(\begin{array}{lll}6 & 13\end{array}\right)$ reflection is $\sim 100$ times less than that of $(631)$ and the only (6 3 1) set is participating in the $\mathrm{BZ}$ construction. For $\mathrm{Al}_{5} \mathrm{CuLi}_{3}$, the $\mathrm{BZ}$ filling by electron state is 0.936 , matching the Hume-Rothery criteria. In addition to this FS-BZ plane contact, it is also necessary to consider the FS overlap with the inner zones, such as the $\left(\begin{array}{lll}5 & 0 & 3\end{array}\right)$-dodecahedron and rhombic triacontahedron formed by $\left(\begin{array}{ll}5 & 3\end{array} 2\right)$ and $\left(\begin{array}{l}6 \\ 0\end{array}\right.$ 0) planes, which should give a significant contribution to the reduction of the electronic energy.

Our next example of approximants-the phase $\mathrm{Au}_{15} \mathrm{Cd}_{23} \mathrm{Zn}_{11}-\mathrm{cl} 146$ was recently found [14] with the valence electron number $z=1.694$. For this $z$ value, the $2 k_{F}$ position is close to $\left(\begin{array}{lll}5 & 3 & 2\end{array}\right)$ and slightly overlaps the $\left(\begin{array}{llll}5 & 0 & 3\end{array}\right)$ and $\left(\begin{array}{llll}6 & 0 & 0\end{array}\right)$ reflections, as shown in Figure 3b. The resulting BZ (Figure $3 b$, lower-right) is completely filled by electron states. It should be noted that BZ construction for this structure was reported (see [14], Figure 34), however, the BRIZ program gives a more accurate construction. Interestingly, for this phase with $\mathrm{z} \approx 1.7$ there are no other diffraction peaks with comparably high intensity, in contrast to the phases with higher $\mathrm{z}$ values. 

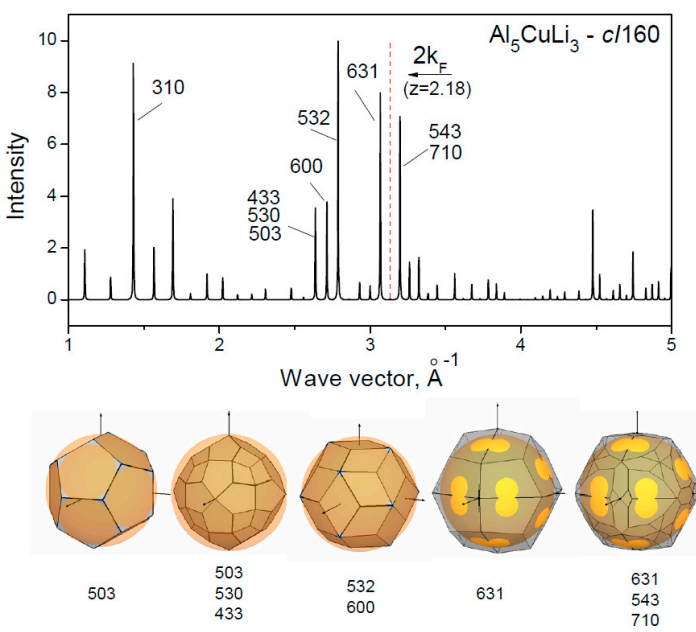

(a)

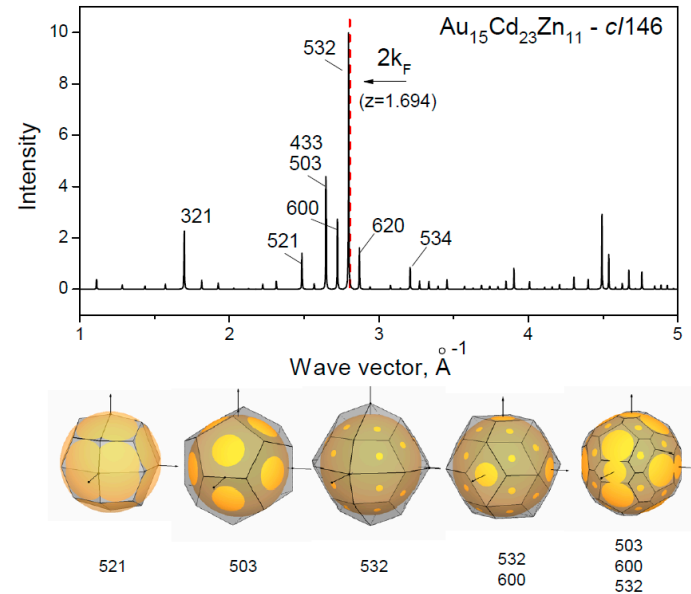

(b)

Figure 3. Calculated diffraction patterns for quasicrystal approximants (upper panel) and corresponding Brillouin-Jones zones with the Fermi spheres (lower panel). The position of $2 k_{F}$ for a given valence electron number per atom $z$ and the hkl indices of the planes used for the BZ construction are indicated on the diffraction patterns. (a) $\mathrm{Al}_{5} \mathrm{CuLi}_{3}-c I 160$; and (b) $\mathrm{Au}_{15} \mathrm{Cd}_{23} \mathrm{Zn}_{11}-c I 146$. Structural data and FS-BZ evaluation data are given in Table 1.

\subsection{Similar Structural Features of Intermetallics with Giant Unit Cells and Approximants}

The complex intermetallic phases discussed in the present paper have similar local atomic arrangement based on tetrahedrally-packed Frank-Kasper polyhedra. Relations of atomic configurations organized in different structural types lead to relations in the form of Brillouin-Jones zones. Examples of such similarity provide the $\mathrm{BZ}$ constructions for $\mathrm{Mg}_{28} \mathrm{Al}_{45}-c I 1168$ and $\mathrm{Al}_{30} \mathrm{Mg}_{40} \mathrm{Zn}_{30}$-cI162 shown in Figures $1 b$ and $2 b$ (lower-right). Diffraction plain sets for BZ construction contacting the FS are as follows:

$\begin{array}{llll}\mathrm{Mg}_{28} \mathrm{Al}_{45} & (1086) & (1420) & (10102) \\ \mathrm{Al}_{30} \mathrm{Mg}_{40} \mathrm{Zn}_{30} & (543) & (710) & (550)\end{array}$

From the comparison of these sets, it can be concluded that the former cell is related to the latter cell as a $2 \times 2 \times 2$ lattice parameter. In this case the number of atoms would be $162 \times 8=1296$, which can be reduced to 1168 by vacancies. It should be noted that, for $\mathrm{Mg}_{28} \mathrm{Al}_{45}$, a reflection (10 100$)$ expected from this transformation also exists, but has very weak intensity, and for the construction of the BZ, the next reflection (10 10 2) was taken. Formation of a complex supercell by multiplication of 
lattice parameters was demonstrated by a classical example of $\gamma$-brass phase $\mathrm{Cu}_{5} \mathrm{Zn}_{8}$. The structure $c I 52$ is formed from $b c c$ with the $2 \times 2 \times 2$ increase of the lattice parameter and an induction of vacancies with some atomic movement that results in an appearance of additional peaks close to the FS, as was discussed by Jones [12]. Interestingly, the forms of the Brillouin zones with faces (8 80 ) and (1133) for $\mathrm{Cu}_{4} \mathrm{Cd}_{3}$ and $\mathrm{Mg}_{28} \mathrm{Al}_{45}$ are similar (Figure 1) and related to the $\mathrm{BZ}$ for $\gamma$-brass with faces (3 30 ) and (4 11 1), which indicates the structural relationship between all these compounds. Structural presentations of complex intermetallics as multi-fold supercells of basic metallic close-packed lattices were considered in $[25,41]$.

Structural relations of alloy phases that are built with similar short-range clusters are visible by comparison of BZs related to strong diffraction peaks. For $\mathrm{Al}_{5} \mathrm{CuLi}_{3}-\mathrm{CI} 160$ the outer $\mathrm{BZ}$ with faces (5 03 3), (6 0 0), and (5 32 2), shown in Figure 3b (lower-right), represents a polyhedron that looks like the Brillouin-Jones zone of quasicrystals i-AlMgCu and i-AlLiCu constructed from the first strong

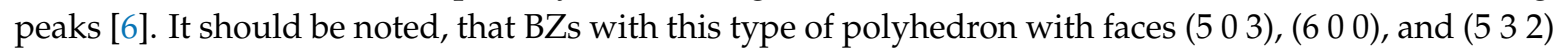
exist for all approximants discussed where the only difference is in the location either outside or inside the FS. In both cases the FS-BZ interaction is significant for the crystal structure energy.

\section{Conclusions}

Structurally-complex alloy phases with tetrahedrally-packed polyhedra of Frank-Kasper type are discussed with reference to the free-electron model of Brillouin zone-Fermi sphere interactions. Electron energy reduction originates through the contact of the FS to the BZ planes, as usually assumed for classical Hume-Rothery phases with relatively simple structures. On their diffraction patterns, complex structures display several peaks of strong intensity that produce Brillouin zone polyhedra enveloped by the FS in contact with some edges or vertices. These FS-BZ configurations should be taken into account for the estimation of electron energy as a convincing reason for stability of such complex intermetallic structures. Complex tetrahedrally-packed intermetallic phases consist of successive atomic building blocks arranged as symmetrical polyhedra. The Brillouin-zone constructions for these structures reveal successive BZ polyhedra with nearly the same form as the atomic clusters in the real space. These effects should be regarded by the theoretical considerations.

Acknowledgments: The authors gratefully acknowledge Dr. Olga Degtyareva for valuable discussions and comments. This work is supported by the program "The Matter under High Pressure" of the Russian Academy of Sciences.

Author Contributions: Valentina F. Degtyareva conceived the project and wrote the paper, and Natalia S. Afonikova analyzed the results, and prepared figures and tables.

Conflicts of Interest: The authors declare no conflict of interest.

\section{References}

1. Frank, F.C.; Kasper, J.S. Complex Alloy structures regarded as sphere packings. I. definitions and basic principles. Acta Cryst. 1958, 11, 184-190. [CrossRef]

2. Mizutani, U. Hume-Rothery Rules for Structurally Complex Alloy Phases; Taylor \& Francis: London, UK, 2010.

3. Steurer, W.; Deloudi, S. Crystallography of Quasicrystals-Concepts, Methods and Structures; Springer Series in Materials Science 126; Springer: Berlin, Germany, 2009.

4. Steurer, W.; Deloudi, S. Fascinating quasicrystals. Acta Cryst. A 2008, 64, 1-11. [CrossRef] [PubMed]

5. Tsai, A.P. Discovery of stable icosahedral quasicrystals: progress in understanding structure and properties. Chem. Soc. Rev. 2013, 42, 5352-5365. [CrossRef] [PubMed]

6. Poon, S.J. Electronic properties of quasicrystals an experimental review. Adv. Phys. 1992, 41, 303-363. [CrossRef]

7. Dubois, J.M. Properties and applications of quasicrystals and complex metallic alloys. Chem. Soc. Rev. 2012, 41, 6760-6777. [CrossRef] [PubMed]

8. Dshemuchadse, J.; Steurer, W. More of the "Fullercages". Z. Anorg. Chem. 2014, 5, 693-700. [CrossRef] 
9. Xie, W.; Cava, R.J.; Miller, G.J. Packing of Russian doll clusters to form a nanometer-scale CsCl-type compound in a Cr-Zn-Sn complex metallic alloy. J. Mater. Chem. C 2017, 5, 7215-7221. [CrossRef]

10. Hume-Rothery, W. Researches on the nature, properties, and condition of formation of intermetallic compounds. J. Inst. Met. 1926, 35, 319-335.

11. Mott, N.F.; Jones, H. The Theory of the Properties of Metals and Alloys; Oxford University Press: London, UK, 1936.

12. Jones, H. The Theory of Brillouin Zones and Electron States in Crystals; North Holland Publ.: Amsterdam, The Netherlands, 1962.

13. Berger, R.F.; Walters, P.L.; Lee, S.; Hoffmann, R. Connecting the chemical and physical viewpoints of what determines structure: From 1-D chains to gamma-brasses. Chem. Rev. 2011, 111, 4522-4545. [CrossRef] [PubMed]

14. Lee, S.; Henderson, R.; Kaminsky, C.; Nelson, Z.; Nguyen, J.; Settje, N.F.; Schmid, J.T.; Feng, J. Pseudo-fivefold diffraction symmetries in tetrahedral packing. Chem. Eur. J. 2013, 19, 10244-10270. [CrossRef] [PubMed]

15. Lee, S.; Leighton, C.; Bates, F.S. Sphericity and symmetry breaking in the formation of Frank-Kasper phases from one component materials. Proc. Natl. Acad. Sci. USA 2014, 111, 17723-17731. [CrossRef] [PubMed]

16. Mizutani, U.; Sato, H. The physics of the hume-rothery electron concentration rule. Crystals 2017, 7, 9. [CrossRef]

17. Degtyareva, V.F. Simple metals at high pressures: The Fermi sphere-Brillouin zone interaction model. Phys-Usp 2006, 49, 369-388. [CrossRef]

18. Degtyareva, V.F. Potassium under pressure: Electronic origin of complex structures. Solid State Sci. 2014, 36, 62-72. [CrossRef]

19. Degtyareva, V.F. Structural simplicity and complexity of compressed calcium: Electronic origin. Acta Crystallogr. B 2014, 70, 423-428. [CrossRef] [PubMed]

20. Degtyareva, V.F.; Afonikova, N.S. Complex structures in the Au-Cd alloy system: Hume-Rothery mechanism as origin. Solid State Sci. 2015, 49, 61-67. [CrossRef]

21. Degtyareva, V.F.; Smirnova, I.S. BRIZ: A visualization program for Brillouin zone-Fermi sphere configuration. Z. Kristallogr. 2007, 222, 718-721. [CrossRef]

22. Degtyareva, V.F.; Degtyareva, O.; Winzenick, M.; Holzapfel, W.B. Structural transformations in a simple-hexagonal Hg-Sn alloy under pressure. Phys. Rev. B 1999, 59, 6058-6062. [CrossRef]

23. Degtyareva, V.F.; Winzenick, M.; Holzapfel, W.B. Crystal structure of InBi under pressure up to $75 \mathrm{GPa}$. Phys. Rev. B 1998, 57, 4975-4978. [CrossRef]

24. Degtyareva, O.; Degtyareva, V.F.; Porsch, F.; Holzapfel, W.B. Face-centered cubic to tetragonal transitions in in alloys under high pressure. J. Phys.-Condens. Mat. 2001, 13, 7295-7303. [CrossRef]

25. Degtyareva, V.F.; Afonikova, N.S. Simple metal and binary alloy phases based on the face centered cubic structure: Electronic origin of distortions, superlattices and vacancies. Crystals 2017, 7, 34. [CrossRef]

26. Smith, A.P.; Ashcroft, N.W. Rapid convergence of lattice sums and structural integrals in ordered and disordered systems. Phys. Rev. B 1988, 38, 12942-12947. [CrossRef]

27. Heine, V.; Weaire, D. Pseudopotential theory of cohesion and structure. Solid State Phys. 1970, 24, $249-463$.

28. Weaire, D.; Williams, A.R. On the axial ration of simple hexagonal alloys of tin. Phil. Mag. 1969, 19, 1105-1109. [CrossRef]

29. Degtyareva, V.F. Electronic origin of the orthorhombic Cmca structure in compressed elements and binary alloys. Crystals 2013, 3, 419-430. [CrossRef]

30. Pearson, W.B. The Crystal Chemistry and Physics of Metals and Alloys; Wiley: New York, NY, USA, 1972.

31. Samson, $\mathrm{S}$. The crystal structure of the intermetallic compound $\mathrm{Cu}_{4} \mathrm{Cd}_{3}$. Acta Cryst. 1967, $23,586-600$. [CrossRef]

32. Samson, $\mathrm{S}$. The crystal structure of the phase $\beta-\mathrm{Mg}_{2} \mathrm{Al}_{3}$. Acta Cryst. 1965, 19, 401-413. [CrossRef]

33. Feuerbacher, M.; Thomas, C.; Makongo, J.P.A.; Hoffmann, S.; Carrillo-Cabrera, W.; Cardoso, R.; Grin, Y.; Kreiner, G.; Joubert, J.; Schenk, T.; et al. The Samson phase, $\beta-\mathrm{Mg} 2 \mathrm{Al} 3$ revisited. Z. Kristallogr. 2007, 222, 259-288.

34. Bruzzone, G. The Ca-Cd and Ba-Cd systems. Gazz. Chim. Ital. 1972, 102, 234-242.

35. Gomez, C.P.; Lidin, S. Comparative structural study of the disordered $\mathrm{MCd}_{6}$ quasicrystal approximants. Phys. Rev. B 2003, 68, 1-9. [CrossRef]

36. Bergman, G.; Waugh, J.L.; Pauling, L. The crystal structure of the metallic phase $\mathrm{Mg}_{32}(\mathrm{AI}, \mathrm{Zn})_{49}$. Acta Cryst. 1957, 10, 254-259. [CrossRef] 
37. Sato, H.; Takeuchi, T.; Mizutani, U. Identification of the Brillouin zone planes in the Hume-Rothery matching rule and their role in the formation of the pseudogap from ab initio band calculations for the Al-Mg- $\mathrm{Zn}$ 1/1-1/1-1/1 approximant. Phys. Rev. B 2001, 64, 094207. [CrossRef]

38. Guryan, C.A.; Stephens, P.W.; Goldman, A.I.; Gayle, F.W. Structure of icosahedral clusters in cubic $\mathrm{Al}_{5.6} \mathrm{Li}_{2.9} \mathrm{Cu}$. Phys. Rev. B 1988, 37, 8495-8498. [CrossRef]

39. Audier, M.; Pannetier, J.; Leblanc, M.; Janot, C.; Lang, J.M.; Dubost, B. An approach to the structure of quasicrystals: A single crystal X-ray and neutron diffraction study of the $\mathrm{R}-\mathrm{Al}_{5} \mathrm{CuLi}_{3}$ phase. Phys. B Condens Mat 1988, 153, 136-142. [CrossRef]

40. Villars, P.; Cenzual, K. (Eds.) Pauling File Binaries Edition; ASM International: Metal Park, OH, USA, 2002.

41. Dshemuchadse, J.; Yung, D.J.; Steurer, W. Structural building principles of complex face-centered cubic intermetallics. Acta Cryst. B 2011, 67, 269-292. [CrossRef] [PubMed]

(C) 2017 by the authors. Licensee MDPI, Basel, Switzerland. This article is an open access article distributed under the terms and conditions of the Creative Commons Attribution (CC BY) license (http://creativecommons.org/licenses/by/4.0/). 